\title{
Microbial glycans: papers from the 7th biennial Baltic Meeting on Microbial Carbohydrates (BMMC)
}

\author{
Cristina De Castro $\cdot$ Otto Holst · Joseph Lam
}

Published online: 27 September 2017

(C) Springer International Publishing AG 2017

In the years 1988-2001, several meetings dedicated to the chemistry and biochemistry of carbohydrates occurred annually at the Research Center Borstel and the University of Rostock, respectively, Germany. Particularly, both conferences created a platform that encourages presentation of studies performed by young researchers from the above institutions, and by colleagues from other institutions in Eastern Europe that were invited to attend. In early 2001, it was agreed to merge both symposia to form a new meeting termed The Carbohydrate Workshop, which took place annually, alternating in Borstel (some $40 \mathrm{~km}$ north of Hamburg) and Güstrow, a town located $60 \mathrm{~km}$ from Rostock. Each meeting focused on different themes, with those in Borstel $(2002,2004)$ on investigation of natural products and medicinal

C. De Castro $(\bowtie)$

Department of Agricultural Sciences, University of

Napoli, Naples, Italy

e-mail: decastro@unina.it

O. Holst

Division of Structural Biochemistry, Research Center Borstel, Leibniz-Center for Medicine and Biosciences, 23845 Borstel, Germany

e-mail: otto.holst@gmail.com

J. Lam

Department of Molecular Cellular Biology, University of Guelph, Guelph, Canada

e-mail: jlam@uoguelph.ca chemistry of (mostly microbial) carbohydrates, whereas the meetings in Güstrow (2001, 2003) concentrated on synthetic carbohydrate chemistry.

A second platform for the exchange of the latest knowledge in carbohydrate chemistry and biochemistry was the German-Polish-Russian Meeting on Bacterial Carbohydrates, which started in Borstel in Year 2000. In 2004, during the third of such meetings in Poland, it was agreed by all of the organizers to merge The Carbohydrate Workshop and the GermanPolish-Russian Meeting to form the biennial Baltic Meeting on Microbial Carbohydrates (BMMC), which has been successfully realized since then.

The 7th BMMC took place in Güstrow on September 25-29, 2016, and comprised a good number of lectures and poster presentations on various topics in the field of microbial carbohydrates.

Topics covered many aspects of glycobiology, spanning from structural chemistry, biochemistry, synthesis, biosynthesis, activity and protein-carbohydrate interactions. Other aspects completed this rich portfolio by reporting the use of state of art techniques in the study of these molecules or the development of computational tools and databases to support the carbohydrate community. Indeed, the programme included four plenary lectures from acknowledged scientists in the field and left ample space to young investigators, resulting in forty oral communications including the Award of Excellence in Glycoscience the Society of Support of Glycoscience, assigned to 
Dr. Alvaro Mallagaray de Benito. The location of the conference and the overall organization were excellent and acted as catalysts by promoting views exchanges and productive discussions between the scientists.

It was agreed with Springer International Publishing $\mathrm{AG}$ and the Editor-in-Chief, Professor Iain Sutcliffe (Northumbria University, UK), to publish a Special Section in the journal Antonie van Leeuwenhoek comprising original articles on research that was presented at the 7th BMMC. We were very glad that this idea was supported by a good number of colleagues who were very much interested to submit manuscripts to contribute to the special publication. Now, in this issue of Antonie van Leeuwenhoek, the Special Section is published, presenting five articles. Particularly, four of these articles report about the structure or the properties of key compounds of bacterial origin, while one examines viral glycosylation.

LPS are amphiphilic molecules and when their primary structure comprises three domains (lipid A, core, O-chain) they are referred as S-type, otherwise if they present only the first two domains they are termed R-type or lipooligosaccharides (LOS). The lipid A consists in a D-glucosamine disaccharide decorated with long fatty acids and phosphate groups; the core region is a medium size oligosaccharide linked to the lipid $\mathrm{A}$ and further elongated with the O-chain, a polysaccharide distinctive for the bacterium strain analysed. The LPS (or LOS) confers to the bacterial membrane its properties, as permeability and adhesion, thus it plays a key role in the adaptation/ interaction of the bacterium to the environment. For instance, mammalian innate immune system reacts to LPS triggering an inflammatory response, and the entity of the reaction strictly depends on the structure of these molecules.

In their work, Zamlynska et al. (2017) (Fig. 1), determine the structure of the lipid A region of the LPS from an environmental bacterium, Phyllobacterium trifolii, a bacterium placed in the order of Rhizobiales and that can establish symbiosis with trifolium and lupines plants. The structure of the main form of lipid A is peculiar (Fig. 1), each glucosamine unit is replaced with 2,3-diacetamido-2,3-dideoxy-D-glucose and the phosphate at the reducing end of the disaccharide is replaced with a galacturonic unit. In addition, fatty acids include the rare 19:1 with a cyclopropane ring, along with the very long fatty acid

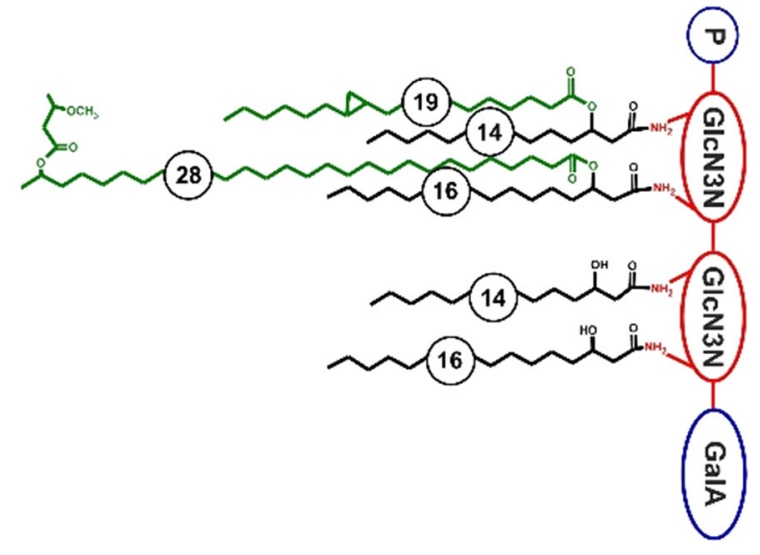

Fig. 1 The structure of lipid A from the LPS of $P$. trifolii

28:0(27-OH). These structural features are discussed in relation to what reported for other bacteria of the same order, and are related to the genetic analysis of the biosynthetic cluster sequenced in the bacterium.

The work from Flaviana Di Lorenzo (2017) (Fig. 2) instead focuses on the lipid A of the obligate marine bacterium Pseudoalteromonas sp. 2A, a strain commensal of Suberites domuncula, a demosponge able to recognize the lipid A moiety of LPS from pathogenic bacteria and to respond producing protecting molecules. In her work, Di Lorenzo discloses that the demosponge-bacterium interaction is possible due to the structural features of the lipid A, that differ from those of lipid A from a pathogenic bacterium, because the phosphorylated glucosamine disaccharide has only 3-5 chains of lauric acid (Fig. 2).

The article from Gleńska-Olender et al. (2017) (Fig. 3), analyses the perception of LPSs from Proteus mirabilis $\mathrm{O} 3$ in healthy humans at different ages, along with patients with rheumatoid arthritis (RA). In this work, the authors have demonstrated that in healthy

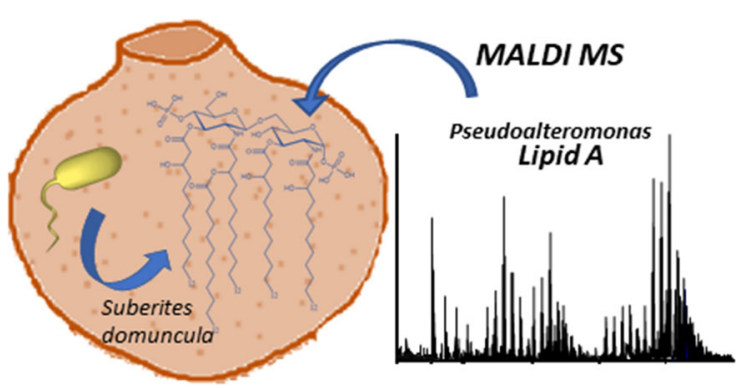

Fig. 2 Lipid A structure from the commensal bacterium Pseudoalteromonas sp. 2A 


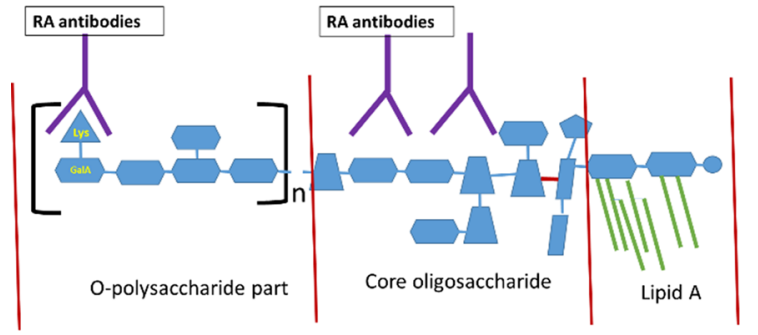

Fig. 3 Interaction of RA antibodies with the O-chain and the core region of the LPS of $P$. mirabilis $\mathrm{O} 3$

volunteers, the titre of antibodies associated to the bacterium increases with the age, probably due to prolonged exposition and the use of the mutant enabled the authors to speculate on which is the epitope recognized from the antibodies. With regard to the RA cohort, authors found an overall increase in the titre of antibodies, even though aspects as specificity, cross-reactivity or nature of the epitope are left to further studies (Fig. 3).

The article from Casillo et al. (2017) focuses on the capsular polysaccharide (CPS) produced from the cold adapted bacterium Colwellia psychrerythraea strain $34 \mathrm{H}$. This extremophile is gaining attention due to the anti-freezing properties and biotechnological potential previously described for the exopolysaccharide. In this paper, the authors investigate a new CPS from the same bacterium, and found that it is made by a trisaccharide repeating unit with only amino-sugar residues and no amino acid (Fig. 4).

Differently from the exopolysaccharide, this CPS has no anti-freeze properties, supporting the functional role played from the amino acid substituents.

Finally, the article from Immacolata Speciale et al. (2017) (Figure 5), reports on a new emerging subject which demonstrates that some virus can glycosylate

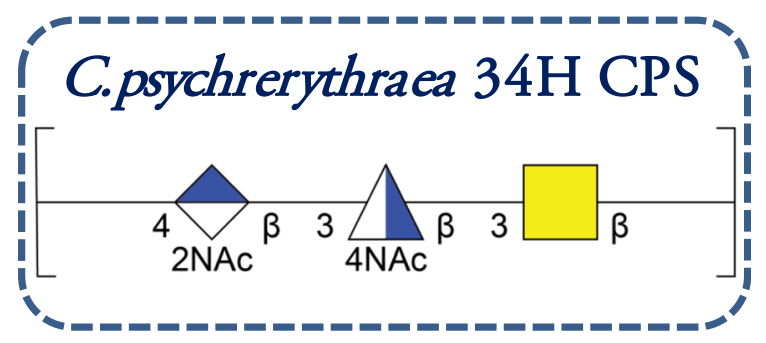

Fig. 4 The structure of the CPS from C. psychrerythraea 34H

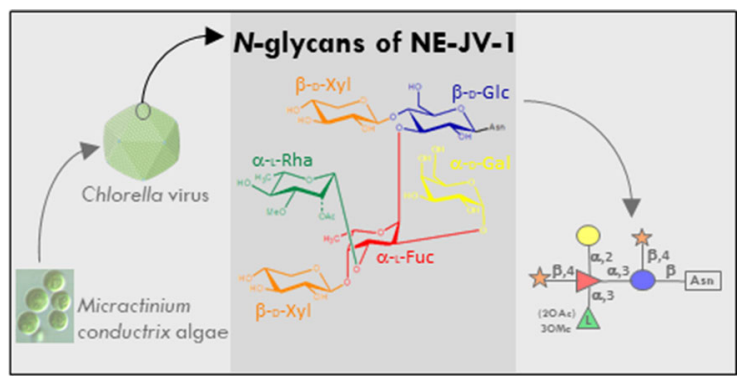

Fig. 5 The complex N-glycan from NE-JV-1 chlorovirus

their protein according to a host-independent process. The structure of the glycan, N-linked to the capsid protein is here described for NE-JV-1, a chlorovirus infecting Micractinium conductrix, a green alga. This $\mathrm{N}$-glycan is a hexasaccharide and its overall architecture matches the conserved core distinctive of chloroviruses.

Concluding, it is our hope that the Special Section will be of support to many researchers in the field, and of inspiration to all those scientists without any previous knowledge on microbial carbohydrates.

Naples, Guelph, Borstel, October 2017

Cristina De Castro

Joseph S Lam

Otto Holst

\section{References}

Casillo A, Ståhle J, Parrilli E et al (2017) Structural characterization of an all-aminosugar-containing capsular polysaccharide from Colwellia psychrerythraea $34 \mathrm{H}$. Antonie van Leeuwenhoek: 1-11

Di Lorenzo F (2017) The lipopolysaccharide lipid A structure from the marine sponge-associated bacterium Pseudoalteromonas sp. 2A. Antonie van Leeuwenhoek: 1-12

Gleńska-Olender J, Durlik K, Konieczna I et al (2017) Detection of human antibodies binding with smooth and rough LPSs from Proteus mirabilis $\mathrm{O}_{3}$ strains $\mathrm{S}_{1959}, \mathrm{R}_{110}, \mathrm{R}_{45}$. Antonie van Leeuwenhoek: 1-9

Speciale I, Agarkova I, Duncan, GA et al (2017) Structure of the $\mathrm{N}$-glycans from the chlorovirus NE-JV-1. Antonie van Leeuwenhoek: 1-9

Zamlynska K, Komaniecka I, Zebracki K et al (2017) Studies on lipid A isolated from Phyllobacterium trifolii $\mathrm{PETP}^{\mathrm{T}}{ }^{\mathrm{T}}$ lipopolysaccharide. Antonie van Leeuwenhoek: 1-21 